\title{
Determination of texture by infrared spectroscopy in titanium oxide-anatase thin films
}

\author{
Carlos Pecharromán ${ }^{\mathrm{a})}$ \\ Instituto de Ciencia de Materiales de Madrid, CSIC, Cantoblanco, 28049 Madrid, Spain \\ Francisco Gracía, Juan P. Holgado, Manuel Ocaña, and Agustín R. González-Elipe \\ Instituto de Ciencia de Materiales de Sevilla (CSIC-University Sevilla), Avda Américo Vespucio s/n, \\ 41092 Sevilla, Spain \\ J. Bassas \\ Serveis Cientificotècnics de la U.B., Solé i Sabarís, 1-3, 08028 Barcelona, Spain \\ J. Santiso and A. Figueras \\ Institut de Ciència de Materials de Barcelona-CSIC, 08193 Bellaterra, Spain
}

(Received 29 May 2002; accepted 21 January 2003)

\begin{abstract}
A theoretical model to determine the effective dielectric tensor of heterogeneous materials composed by anisotropic microcrystallites has been introduced to explain the infrared spectral features of textured thin films of uniaxial materials as the function of a textural parameter. This theoretical treatment is able to satisfactorily reproduce the experimental absorbance spectra of $\mathrm{TiO}_{2}$-anatase thin films chosen as a model system. Comparison of texture data obtained from infrared spectroscopy and x-ray diffraction are in good agreement which support the validity of the proposed model. (C) 2003 American Institute of Physics. [DOI: 10.1063/1.1560858]
\end{abstract}

\section{INTRODUCTION}

Infrared absorption/reflection infrared (IR) spectroscopy on thin films is commonly used as an analytic technique to identify the nature of the deposited materials. When done at normal incidence, transverse optical phonons of isotropic compounds can be detected as absorption maxima, while at grazing angles and perpendicular polarization these modes vanish substituted by longitudinal optical phonons. This effect, firstly reported by Berreman, ${ }^{1}$ has been widely used to experimentally determine the longitudinal frequencies of several cubic substances. ${ }^{2,3}$ However, in the case of anisotropic samples, this effect is only verified under very restrictive conditions, which depend on the orientation of the optical axes of the layer. In fact, in anisotropic layers pure longitudinal or transverse phonons ${ }^{4}$ only propagates in very specific directions, related with the optical axes of the crystal.

Most thin film growth techniques produce polycrystalline films. The physical properties of these layers can be considered isotropic (at a length scale larger than the crystallite size) even or anisotropic materials if the crystallites are randomly distributed and oriented. The optical properties of highly diluted composites of these materials can be calculated by an effective dielectric constant approximation ${ }^{5}$ and the original Berreman's formulation ${ }^{1}$ can still be used. However, this approximation fails in anisotropic substances when the preparation procedure introduces a preferential texture, i.e., when the film depicts a preferential orientation of some of their crystal planes. In these cases, thin films behave as anisotropic media.

\footnotetext{
a) Author to whom correspondence should be addressed; electronic mail: cpg@icmm.csic.es
}

Preferential texturing of thin films is easily identified by $\mathrm{x}$-ray diffraction (XRD) in a Bragg-Brentano configuration because the diffraction peaks of the planes preferentially oriented according to the thin film surface present an enhanced intensity.

There is some previous publications which tried to link the effective ${ }^{6}$ and average $\mathrm{e}^{7,8}$ dielectric constant with textural parameters, but there is not a systematic treatment of this subject. In a previous publication, we used a qualitative approach by Fourier transform IR spectroscopy (FT-IR) to deduce preferential orientation phenomena in $\mathrm{Fe}_{2} \mathrm{O}_{3}$ pressed pellets ${ }^{9}$ and thin films. ${ }^{10}$ In the present article we introduce a theoretical treatment of FT-IR spectra of thin films that enables us to gain information about the preferential orientation of crystal planes. For a sake of simplicity we have restrict the range of application of this model to uniaxial compounds, i.e., those that crystallize into the tetragonal, rhombohedral, and hexagonal systems, but the extension to biaxial ones is straightforward. To verify the possibilities of the model, characterization experiments by FT-IR, with polarized and unpolarized light, and XRD of $\mathrm{TiO}_{2}$ anatase thin films have been carried out. The good concordance of the results by the two techniques, indicating the preferential orientation of a given type of crystal planes, has confirmed the predictions of the model and open the possibility of systematically using FT-IR for proving preferential orientation phenomena in thin films.

\section{EXPERIMENT}

Very compact and dense $\mathrm{TiO}_{2}$ thin films have been prepared by ion beam induced chemical vapor deposition. A thorough description of this procedure can be found in a previous publication. ${ }^{11}$ Briefly, it consists of inducing the decomposition of a volatile precursor of the metal (in this 
case Ti) with a beam of accelerated $\mathrm{O}_{2}^{+}$ions. Here, Ti isopropoxide has been used as volatile precursor of $\mathrm{Ti}$ and the beam energy and current at the substrate position were 400 $\mathrm{eV}$ and $100 \mu \mathrm{A} \mathrm{cm}{ }^{-2}$, respectively. They were grown at room temperature on a flat $\mathrm{Si}(100)$ substrate. The film thickness was $450 \mathrm{~nm}$. After preparation, the films were amorphous but they crystallized into the anatase structure of $\mathrm{TiO}_{2}$ after annealing in air at $T \geqslant 300^{\circ} \mathrm{C}$. Samples annealed at 300 and $600{ }^{\circ} \mathrm{C}$ were studied by XRD and FT-IR (hereafter these samples will be called $\mathrm{TiO}_{2}-300$ and $\mathrm{TiO}_{2}-600$ ).

$\mathrm{X}$-ray powder diffraction patterns were obtained in a Bragg-Brentano $\theta / 2 \theta$ diffractometer, Siemens D500; a divergence slit of $1^{\circ}$ was used, resulting on an incident beam of $3.8 \mathrm{~mm}$ at the sample position. The $\mathrm{Cu} K \alpha$ radiation was used as excitation source at a working power of $40 \mathrm{kV} \times 30$ $\mathrm{mA}$.

FT-IR spectra with unpolarized light were recorded from 200 to $1000 \mathrm{~cm}^{-1}$ in the transmission mode in a Nicolet 510 Fourier transform spectrometer. FT-IR spectra with polarized light were recorded $400-1000 \mathrm{~cm}^{-1}$ in a Nicolet 20 SXC by using a wire $\mathrm{KBr}$ polarizer. The angle between the beam and the normal to the thin film surface was changed for different measurements.

\section{THEORETICAL PROCEDURE}

\section{A. Effective medium approximation for dielectric composites}

In contrast to isotropic polycrystalline materials, the optical properties of dense anisotropic solids can be substantially different from those of single crystals, as a consequence of the decisive role of the orientation of the crystallites in these materials. Thus, a sort of average of the optical properties of the single crystallites along different directions of the dielectric tensor should be expected in macroscopic samples. In order to properly calculate the properties of these materials, an effective medium theory has been introduced, based on an effective medium formalism developed by the authors in some previous publications. ${ }^{9,12}$ This approach, which can be considered as a generalization of several others models previously presented, ${ }^{5,13,14}$ was formulated for isotropic and anisotropic particles randomly distributed into a heterogeneous material, in such a way that the whole composite presents an isotropic macroscopic behavior. In this context, it was assumed for the isotropic case that the average electric field in the vicinity of the particles was constant, homogeneous and identical to the average electric field $\langle E\rangle_{\mathbf{E}_{0}}$, (effective medium theory). In addition, it is supposed that particle shape is ellipsoidal, so that the electric field inside this particles is also constant and can be easily calculated. However, when crystallites present textured distributions, the composite may be anisotropic and in this case the electric field inside of a ellipsoidal article is no longer isotropic nor homogeneous even if the external field is assumed to be homogeneous. However, a detailed calculation shows (Appendix A) that although the heterogeneous medium can display an anisotropic behavior, each component of the macroscopic effective dielectric tensor along its optical axes can be reasonably approximated by an effective medium approximation. Thus, according to Refs. 12 and 9, and the conclusions obtained in Appendix A, the general equation which determines the effective dielectric constant of a heterogeneous composite formed by two anisotropic phases (labeled by subindex " $p$ " and " $m$ ") along the direction $l$, is given by:

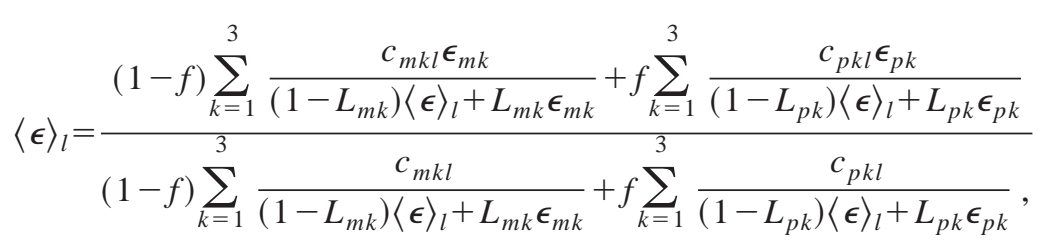

where subindex $k$ refers to the axes $(x, y$ and $z$ ), which for spheroidal shapes, are restricted to $x$ and $z ; f$ is the filling factor, $L_{m k}$ and $L_{p k}$ the depolarization factors of the matrix and particle spheroids along the $k$ axis, and $c_{m k l}$ and $c_{p k l}$ the average of the projection of a unit vector parallel to the optic axis onto the applied electric field.

\section{B. Application of the effective medium approximation to dense materials}

In the case of a dense aggregate, i.e., $f=1$, the $m$ phase is absent so that it can be dropped from Eq. (1). Then the effective dielectric constant along the $l$ axis notably simplify into:

$$
\sum_{k=1}^{3} \frac{c_{p k l}\left(\epsilon_{p k}-\langle\epsilon\rangle_{l}\right)}{\left(1-L_{p k}\right)\langle\epsilon\rangle_{l}+L_{p k} \epsilon_{p k}}=0 .
$$

It should be noted that these expressions of the effective dielectric tensor components depend on geometrical factors of the crystalline grains, i.e., the grain shape through $L_{p k}$ and on the average orientation given by $c_{p k l}$. In this sense, any texture effect on the IR spectra should be well described by these coefficients. In order to greatly simplify the notation, we have assumed that crystallites are optically uniaxial so that each crystallite has its own optical axis (denoted by " $o$ " and " $e$ " for ordinary and extraordinary rays), which defines an angle $\theta$ with the $z$ axis of the layer. If cylindrical symmetry is assumed for the thin layer, it must become optically uniaxial, being $\langle\epsilon\rangle_{z}$ the dielectric constant along the $z$ axis 
(a)

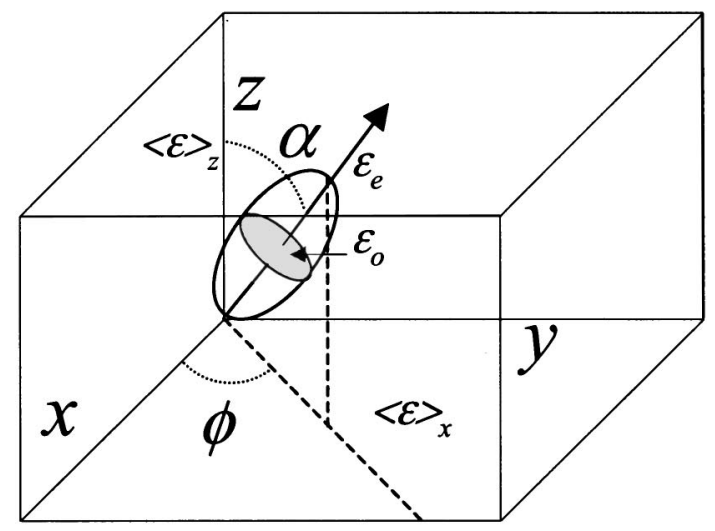

(b)

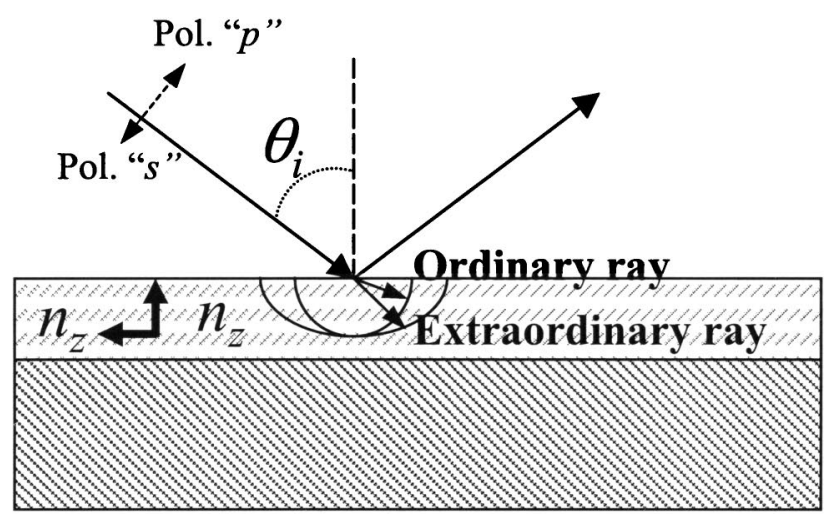

FIG. 1. (a) Geometry of an ellipsoidal particle in the layer frame; (b) geometry of a plane thick layer.

and $\langle\epsilon\rangle_{x}$ that corresponding to the $x-y$ plane. The relationships between optical axes of the composite, $x$ and $z$, with those of the crystallite can be deduced from Fig. 1(a). Using the definition of $c_{p k l}$ enclosed in Eq. (1) as it appears in Appendix A, we get the analytic expression of these geometric coefficients

$$
\begin{aligned}
& c_{\mathrm{pex}}=1 / 4 \pi \int \sin ^{2}(\theta) \gamma_{\mathrm{pe}}(\theta) d \Omega, \\
& c_{\mathrm{pez}}=1 / 4 \pi \int \cos ^{2}(\theta) \gamma_{\mathrm{pe}}(\theta) d \Omega, \\
& c_{\mathrm{pox}}=1 / 4 \pi \int \sin ^{2}(\theta) \gamma_{\mathrm{po}}(\theta) d \Omega, \\
& c_{\mathrm{poz}}=1 / 4 \pi \int \cos ^{2}(\theta) \gamma_{\mathrm{po}}(\theta) d \Omega,
\end{aligned}
$$

where $d \Omega$ is the solid angle differential, and $\gamma_{\mathrm{pe}}$ and $\gamma_{\mathrm{po}}$ are the probability density function corresponding to the angular orientation of the extraordinary and ordinary optical axis. It should be noted that the terms $\sin ^{2}(\theta)$ and $\cos ^{2}(\theta)$ in Eqs. (3)-(6) are obtained as a result of the projection of each component of the dielectric tensor to $\gamma_{k l, \mathbf{E}_{0}}(\theta)$, i.e., the den- sity function corresponding to the probability of finding that the $l$ axis of the ellipsoid makes an angle $\theta$ with $\mathbf{E}_{0}$. Due to the fact that axial symmetry has been assumed along the perpendicular to the sample surface, then, $\gamma_{\mathrm{pe}}$ and $\gamma_{\mathrm{po}}$ only depend on the angle $\theta$. Moreover, in uniaxial substances the ordinary axes are degenerated into a plane, then

$$
\frac{2 \int \gamma_{\mathrm{po}} d \Omega+\int \gamma_{\mathrm{pe}} d \Omega}{4 \pi}=1 .
$$

Thus we get the following relationships between the four different coefficients:

$$
\left\{\begin{array}{l}
2 c_{\mathrm{pox}}+c_{\mathrm{pex}}=1 \\
2 c_{\mathrm{poz}}+c_{\mathrm{pez}}=1 \\
2 c_{\mathrm{pox}}+c_{\mathrm{poz}}=1 \\
2 c_{\mathrm{pex}}+c_{\mathrm{pez}}=1 .
\end{array}\right.
$$

If we consider that $c_{\text {pez }}$ can take any value from 0 to 1 , then Eqs. (8) impose the limiting conditions for the rest of the $c$ coefficients

$$
\left\{\begin{array}{l}
c_{\mathrm{pez}}=0 \ldots 1 \\
c_{\mathrm{pex}}=\frac{1-c_{\mathrm{pez}}}{2}=\frac{1}{2} \ldots 0 \\
c_{\mathrm{poz}}=\frac{1-c_{\mathrm{pez}}}{2}=\frac{1}{2} \ldots 0 \\
c_{\mathrm{pox}}=\frac{1+c_{\mathrm{pez}}}{4}=\frac{1}{4} \ldots \frac{1}{2} .
\end{array}\right.
$$

At this point it is possible to simplify Eq. (2) to obtain two expressions for determining the effective dielectric constant along the $x$ and $z$ axes

$$
\begin{gathered}
\frac{\left(1+c_{\mathrm{pez}}\right)}{2} \frac{\left(\langle\epsilon\rangle_{x}-\epsilon_{\mathrm{po}}\right)}{\left(1-L_{\mathrm{po}}\right)\langle\epsilon\rangle_{x}+L_{\mathrm{po}} \epsilon_{\mathrm{po}}} \\
+\frac{\left(1-c_{\mathrm{pez}}\right)}{2} \frac{\left(\langle\epsilon\rangle_{x}-\epsilon_{\mathrm{pe}}\right)}{2 L_{\mathrm{po}}\langle\epsilon\rangle_{x}+\left(1-2 L_{\mathrm{po}}\right) \epsilon_{\mathrm{pe}}}=0, \\
\left(1-c_{\mathrm{pez}}\right) \frac{\left(\langle\epsilon\rangle_{z}-\epsilon_{\mathrm{po}}\right)}{\left(1-L_{\mathrm{po}}\right)\langle\epsilon\rangle_{z}+L_{\mathrm{po}} \epsilon_{\mathrm{po}}} \\
+c_{\mathrm{pez}} \frac{\left(\langle\epsilon\rangle_{z}-\epsilon_{\mathrm{pe}}\right)}{2 L_{\mathrm{po}}\langle\epsilon\rangle_{z}+\left(1-2 L_{\mathrm{po}}\right) \epsilon_{\mathrm{pe}}}=0 .
\end{gathered}
$$

These expressions look very similar to that obtained in the well known Bruggemann effective medium theory. ${ }^{15,16}$ That approximation was formulated for heterogeneous media composed by two isotropic phases, being $f$ the volume concentration of one of them. In our model the composite only has one single anisotropic phase but two different components of the dielectric tensor. In this case the role of the phases is replaced with the dielectric tensor components of crystallites. Moreover, the role of the volume concentration, $f$, is assumed in a dense anisotropic composite by two expressions related to the orientation coefficient $c_{\mathrm{pez}}$. It should be noted that Eqs. (10) and (11) are two second degree equations in $\langle\epsilon\rangle_{x}$ and $\langle\epsilon\rangle_{z}$.

$$
\begin{aligned}
& a_{x}\langle\epsilon\rangle_{x}^{2}+\langle\epsilon\rangle_{x}\left(\epsilon_{o} b_{o x}+\epsilon_{e} b_{e x}\right)-\epsilon_{e} \epsilon_{o} c_{x}=0, \\
& a_{z}\langle\epsilon\rangle_{z}^{2}+\langle\epsilon\rangle_{z}\left(\epsilon_{o} b_{o z}+\epsilon_{e} b_{e z}\right)-\epsilon_{e} \epsilon_{o} c_{z}=0 .
\end{aligned}
$$


TABLE I. Effective dielectric constant, anisotropic character, and weight of each kind of phonon (transverse, longitudinal, ordinary, and extraordinary) in the absorption spectrum as a function of the crystallite orientation angle.

\begin{tabular}{lcccccccc}
\hline \hline$\alpha$ & & & & $W\left(\omega_{T O}\right)$ & $W\left(\omega_{L O}\right)$ & $W\left(\omega_{T E}\right)$ & $W\left(\omega_{L E}\right)$ \\
$c_{\mathrm{pez}}$ & $\epsilon_{x}$ & $\epsilon_{z}$ & Anisotropy & $\begin{array}{c}\theta_{i}=0^{\circ} \\
\theta_{i}=90^{\circ}\end{array}$ & $\begin{array}{c}\theta_{i}=0^{\circ} \\
\theta_{i}=90^{\circ}\end{array}$ \\
\hline $90^{\circ}$ & 0 & $\frac{\epsilon_{o}+\epsilon_{e}}{2}$ & $\epsilon_{o}$ & Yes & $1 / 2$ & $1 / 2$ & 1 & 0 \\
$45^{\circ}$ or random & $1 / 3$ & $\frac{2 \epsilon_{o}+\epsilon_{e}}{3}$ & $\frac{2 \epsilon_{o}+\epsilon_{e}}{3}$ & No & $2 / 3$ & $1 / 3$ & $2 / 3$ & $1 / 3$ \\
$0^{\circ}$ & 1 & $\epsilon_{o}$ & $\epsilon_{e}$ & Yes & 1 & 0 & 0 & 1 \\
\hline \hline
\end{tabular}

The coefficients of these equations are given by

$$
\begin{aligned}
& \left\{\begin{array}{l}
a_{x}=1+L_{p}+c_{\mathrm{pez}}\left(-1+3 L_{p}\right) \\
b_{o x}=-L_{p}-3 c_{\mathrm{pez}} L_{p} \\
b_{e x}=-L_{p}+2 c_{\mathrm{pez}}-3 c_{\mathrm{pez}} L_{p} \\
c_{x}=1-L_{p}+c_{\mathrm{pez}}\left(1-3 L_{p}\right),
\end{array}\right. \\
& \left\{\begin{array}{l}
a_{z}=2 L_{p}+c_{\mathrm{pez}}\left(1-3 L_{p}\right) \\
b_{o z}=-2 L_{p}+3 c_{\mathrm{pez}} L_{p} \\
b_{e z}=1-2 L_{p}-2 c_{\mathrm{pez}}+3 c_{\mathrm{pez}} L_{p} \\
c_{z}=1-2 L_{p}-c_{\mathrm{pez}}\left(1-3 L_{p}\right) .
\end{array}\right.
\end{aligned}
$$

To illustrate the relationship between these coefficients with textural parameters given by $\gamma_{\mathrm{pe}}$, [Eqs. (3)-(6)] let us consider two extreme cases: (i) a layer made by crystallites randomly oriented and (ii) a layer formed by crystallites totally oriented towards a specific orientation, which describes an angle $\alpha$ with the layer normal.

In the first case $\gamma_{\mathrm{pe}}(\theta)$ is a constant independent of $\theta$, so that $c_{\mathrm{pez}}=c_{\mathrm{pex}}=1 / 3$ and according to Eqs. (10) and (11) the heterogeneous medium presents an isotropic behavior $\left(\langle\epsilon\rangle_{x}\right.$ $\left.=\langle\epsilon\rangle_{z}\right)$. This results coincides with the isotropic previous models. ${ }^{13,14}$ In the second case, the function $\gamma_{\mathrm{pe}}(\theta)$ can be written as a Dirac's delta

$$
\gamma_{\mathrm{pe}}(\theta)=g \cdot \delta(\theta-\alpha),
$$

where $g$ is the normalization constant. By using Eq. (4) we get:

$$
\begin{aligned}
c_{\mathrm{pez}} & =g \int_{0}^{\pi / 2} \cos ^{2}(\theta) \sin (\theta) \delta(\theta-\alpha) d \theta \\
& =g \cos ^{2}(\alpha) \sin (\alpha) .
\end{aligned}
$$

The coefficient $c_{\text {pex }}$ can be obtained operating in the same way with Eq. (3). Then, by using Eq. (8), it is possible to determine $g$ to get the final expression of $c_{\mathrm{pez}}$ as a function of $\alpha$.

$$
c_{\mathrm{pez}}(\alpha)=\frac{\cos ^{2}(\alpha)}{1+\sin ^{2}(\alpha)} .
$$

Equations (10) and (11) in combination with Eq. (18) allow us to relate the optical anisotropy of the polycrystalline material with the angle of preferential orientation. Assuming a quasispherical particular shape $\left(L_{p}=1 / 3\right)$, we can state that different anisotropy schemes (Table I) could appear in a polycrystalline anisotropic material as a function of the orientation coefficient $c_{\mathrm{pez}}$.
If all the crystallographic axes of the crystallites are perpendicularly oriented to the normal of the surface sample, i.e., $\alpha=90^{\circ}$ then, by using Eq. (18), it results that $c_{\text {pez }}=0$. The substitution of this value in Eqs. (10) and (11) determines that $\left\langle\epsilon_{x}\right\rangle$, the dielectric tensor coefficient corresponding to the plane parallel to the surface sample, is equal to the arithmetic mean value of ordinary and extraordinary components of crystallites ( $\epsilon_{o}$ and $\epsilon_{e}$ ), while the $\left\langle\epsilon_{z}\right\rangle$ component is identical to the ordinary one of the microcrystals $\left(\epsilon_{o}\right)$. Thus, the absorbance IR spectrum detected for the sample under normal incidence will be a combination of transverse modes of the ordinary and extraordinary rays. However by tilting the sample, longitudinal modes of the ordinary ray begin to appear, while longitudinal modes of the extraordinary component remain absent.

On the contrary, if all the crystallites axes are perpendicular to the sample surface we get $\alpha=0^{\circ}$, and $c_{\mathrm{pez}}=1$. This geometric configuration is identical, from an optical point of view, to that of a single (anisotropic) crystal with its optical axis parallel to the normal to the crystal surface. Under these circumstances, we must observe the transverse ordinary modes by IR absorption spectroscopy at normal incidence, while only the longitudinal extraordinary modes must be detected at grazing angles.

Finally, if crystallites are randomly oriented, according to Eq. (4), it results that $c_{\mathrm{pez}}=1 / 3$, a value which coincides with that of crystallite axes oriented $45^{\circ}$ with respect to the normal to the plane. Under these conditions, Eqs. (10) and (11) determine that the whole sample behaves as an isotropic material, being identical to all the components of the dielectric tensor. It should be noted that this angle is different from the so called "magic angle" $\left[\cos ^{2}\left(\theta_{M}\right)=1 / 3\right.$ or $\theta_{M}$ $\left.=54^{\circ} 44^{\prime}\right]$ which corresponds to an angular average of the considered magnitude ${ }^{7,8}$ (permittivity, refractive index, reflectivity, etc). The discrepancy in our case is due to the fact that the calculation of effective dielectric constant takes into account the projection of the internal field of the particles (which in our case satisfies the conditions of magic angle) over the $x y$ plane and the $z$ axis, instead of the average permittivity tensor.

These results are the limit cases that can appear in a dense anisotropic polycrystalline material. Thus, they can be used as a quick test to detect texture in polycrystalline thin layers measured by IR absorption spectra at normal and grazing angles. 


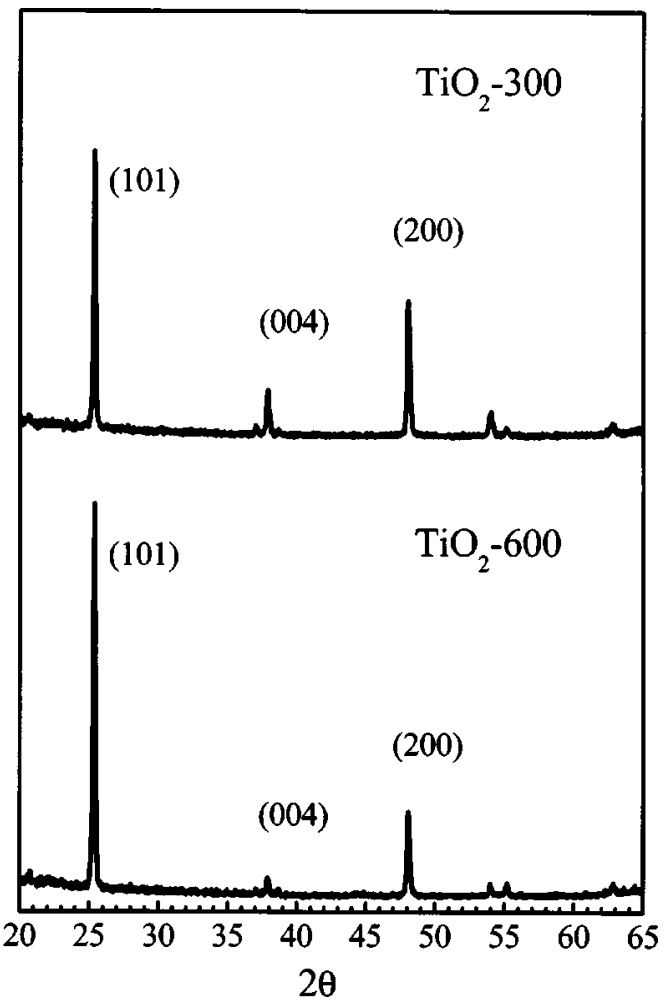

FIG. 2. XRD of anatase thin films prepared at 300 and $600{ }^{\circ} \mathrm{C}$.

However, a quantitative evaluation of the optical properties of anisotropic thin layers is somehow more complicated ${ }^{17}$ and requires the use of the $4 \times 4$ transfer matrix formulation. ${ }^{18,19}$ This method has been recently employed in ellipsometry in order to determine the optical constants of single crystal anisotropic layers..$^{20-22}$ We have used the explicit formulation given by Schubert ${ }^{23}$ as it appears in Appendix B.

Once the full set of equations is implemented we get a calculation procedure which is able to estimate the theoretical spectra of dense heterogeneous anisotropic thin layers, where anisotropy is a consequence of preferential orientation of its crystallites. In order to obtain its theoretical spectrum the following data are necessary: thickness of the layer, incidence angle and polarization degree of incident beam, refractive index of microparticles, usually measured on single crystals, approximate shape of crystallites $\left(L_{p}\right)$, and orientation degree $\left(c_{\text {pez }}\right)$.

It is clear that a dense material composed by anisotropic grains can present a rich variety of different spectra especially if $\epsilon_{o}$ and $\epsilon_{e}$ have large differences in a wide spectral range.

\section{RESULTS}

\section{A. Textural analysis of $\mathrm{TiO}_{2}$ thin films by XRD}

The XRD patterns corresponding to the two samples studied here (i.e., $\mathrm{TiO}_{2}-300$ and $\mathrm{TiO}_{2}-600$ ) are shown in Fig. 2 . The two diagrams show a diffraction pattern typical of that of anatase. For a comparative analysis of the two diagrams,
TABLE II. Intensities, normalization factors, and corrected intensities of the diffraction peaks of anatase thin films.

\begin{tabular}{|c|c|c|c|c|c|c|c|c|c|}
\hline \multirow[b]{3}{*}{$h k l$} & \multirow[b]{3}{*}{$2 \theta$} & \multicolumn{4}{|c|}{$\mathrm{TiO}_{2}-300$} & \multicolumn{4}{|c|}{$\mathrm{TiO}_{2}-600$} \\
\hline & & \multicolumn{2}{|c|}{$(\tau=0.45 \mu \mathrm{r}$} & \multicolumn{2}{|c|}{, $\left.2 \theta_{\mathrm{L}}=42.0^{\circ}\right)$} & \multicolumn{2}{|c|}{$(\tau=0.32$} & \multicolumn{2}{|c|}{$\left.2 \theta_{L}=49.4^{\circ}\right)$} \\
\hline & & $C_{h k l}$ & $T^{\mathrm{obs}}$ & $I^{c}$ & $R^{c}$ & $C_{h k l}$ & $I^{\mathrm{obs}}$ & $I^{c}$ & $R^{c}$ \\
\hline 101 & 25.308 & 1.000 & 53.0 & 1214 & 1.000 & 1.000 & 73.0 & 2352 & 1.000 \\
\hline 004 & 37.791 & 1.000 & 11.0 & 252 & 0.208 & 1.000 & 4.0 & 129 & 0.055 \\
\hline 200 & 48.047 & 1.137 & 34.0 & 886 & 0.730 & 1.000 & 21.0 & 677 & 0.288 \\
\hline 105 & 53.885 & 1.265 & 7.5 & 217 & 0.179 & 1.084 & 2.5 & 87 & 0.037 \\
\hline 211 & 55.073 & 1.291 & 1.8 & 53 & 0.044 & 1.106 & 3.3 & 118 & 0.050 \\
\hline 204 & 62.692 & 1.452 & 3.0 & 99 & 0.082 & 1.245 & 2.5 & 132 & 0.056 \\
\hline
\end{tabular}

the observed integrated intensities $\left(I^{\mathrm{obs}}\right)$ of the main reflections are corrected by taking into account both layer thickness and sample size:

$$
\frac{I^{c}}{I^{\mathrm{obs}}}=\frac{C_{h k l}}{2 \mu \tau},
$$

where $C_{h k l}$ is a normalization factor calculated as $C_{h k l}$ $=\sin \theta / \sin \theta_{L}$ (if $\theta>\theta_{L}$ ); and $C_{h k l}=1$ (if $\theta<\theta_{L}$ ); the incident angle cutoff, $\theta_{L}$, depends on the sample size and the incident beam cross section $\left(\theta_{L}=21.0^{\circ}\right.$ and $24.7^{\circ}$ for the $\mathrm{TiO}_{2}-300$ and $\mathrm{TiO}_{2}-600$ samples respectively); $\tau$, the layer thickness and the absorption coefficient for $\mathrm{TiO}_{2}$-anatase, is $\mu=0.0485 \mu \mathrm{m}^{-1}$.

Observed and corrected intensities are indicated in Table II, as well as the relative corrected intensities $\left(R^{c}=I^{c} / I_{101}^{c}\right)$ referred to the 101 reflection.

The degree of orientation for a given diffracting plane can be calculated considering the deviation from the relative corrected intensities with respect to the same planes in a random distributed anatase. The used coefficient $Q_{h k l}$ is

$$
Q_{h k l}=\frac{R_{h k l}^{c}}{\beta_{h k l}}-1,
$$

where $\beta$ means the relative integrated intensity of the $h k l$ reflection with respect the 101 one $(100 \%)$ when the material is randomly oriented.

The relative density of planes of a given orientation, $N_{h k l}$, can be also estimated from relative corrected intensities, using the normalized expression

$$
N_{h k l}=\frac{R_{h k l}^{c} / \beta_{h k l}}{\sum_{N_{m x}}^{i=1} R_{c}^{i} / \beta_{i}},
$$

where the summation extends over all the considered reflections along the diffraction pattern. For the present work, we have considered $N_{m x}=6$ different reflections, as it appears in Table II. Consequently, $N_{h k l}=1 / 6$ would correspond to a random orientation and $N_{h k l}>1 / 6$ indicates that $(h k l)$ planes are preferentially oriented parallel to the sample surface, the higher the value the stronger the orientation.

The $Q_{h k l}$ and $N_{h k l}$ values obtained for both samples studied are summarized in the Table III. As observed, the most significant difference between the two samples regarding the relative orientation of their crystallographic planes corresponds to the (101) plane. In fact, this plane has $N_{h k l}$ 
TABLE III. Texture parameters of the $\mathrm{TiO}_{2}$ samples.

\begin{tabular}{lcccccccc}
\hline \hline & & \multicolumn{3}{c}{$\mathrm{TiO}_{2}-300$} & & \multicolumn{3}{c}{$\mathrm{TiO}_{2}-600$} \\
\cline { 3 - 5 } \cline { 7 - 9 }$h k l$ & $\beta_{h k l}$ & $R_{h k l}^{c}$ & $Q_{k h l}$ & $N_{h k l}$ & & $R_{h k l}^{c}$ & $Q_{k h l}$ & $N_{h k l}$ \\
\hline 101 & 1.000 & 1.000 & 0.00 & 0.16 & & 1.000 & 0.00 & 0.32 \\
004 & 0.206 & 0.208 & 0.01 & 0.16 & & 0.055 & -0.73 & 0.09 \\
200 & 0.290 & 0.730 & 1.52 & 0.40 & & 0.288 & -0.01 & 0.32 \\
105 & 0.188 & 0.179 & -0.05 & 0.15 & & 0.037 & -0.80 & 0.06 \\
211 & 0.187 & 0.044 & -0.76 & 0.04 & & 0.050 & -0.73 & 0.09 \\
204 & 0.148 & 0.082 & -0.45 & 0.09 & 0.056 & -0.62 & 0.12 \\
\hline \hline
\end{tabular}

values of 0.16 and 0.32 in the $\mathrm{TiO}_{2}-300$ and $\mathrm{TiO}_{2}-600$ samples, respectively. These values indicate that while this family of planes is randomly oriented in the first sample, it presents a clear preferential orientation parallel to the thin film surface in the second sample. A smaller change (i.e., from 0.40 to 0.32 ) in the $N_{h k l}$ value is also found for the (200) planes when comparing the two samples, although in the two cases the parameter was almost double that of 0.16 , the typical value of a randomly oriented sample. This means that in the two samples the (200) family of planes is preferentially oriented parallel to the thin film surface and that there are no significant differences between them in the relative orientation degree. For the (004) planes the situation is the opposite since these planes lose orientation degree when comparing the $\mathrm{TiO}_{2}$-300 with the $\mathrm{TiO}_{2}-600$ samples (i.e., $N_{h k l}$ values of 0.16 and 0.09 ). The other reflections whose intensities have been considered for the calculations are very small and do not present any preferential orientation in the two samples (i.e., $N_{h k l}$ values smaller than 0.16 in all cases). Considering these data, we will refer to the (001) planes for the use of FT-IR data to account for preferential orientation phenomena.

\section{B. FT-IR analysis of $\mathrm{TiO}_{2}$ thin films}

Figure 3 shows the IR absorption spectra of $\mathrm{TiO}_{2}-300$ and $\mathrm{TiO}_{2}-600$ samples taken at different incident angles. The series of bands appearing in these spectra are attributed to lattice vibrational modes. Their assignment according to the data reported in the literature for anatase ${ }^{24}$ appear in Table IV. The sample $\mathrm{TiO}_{2}-300$ are characterized by two bands at 262 and $435 \mathrm{~cm}^{-1}$ ascribed to the transverse optical phonons. There is also a feature, corresponding to the longitudinal modes in the spectral region from 750 to $830 \mathrm{~cm}^{-1}$, which becomes more prominent at higher incidence angles. The spectrum of sample $\mathrm{TiO}_{2}-600$ displays similar bands at normal incidence. However, the largest difference of these spectra compared with the previous ones is the shape of the band corresponding to longitudinal mode measured at highest incidence angles. In the high temperature samples, a well defined maximum appear at $856 \mathrm{~cm}^{-1}$ for incidence angles larger than $45^{\circ}$.

A proper analysis of the IR spectra at different incidence angles with incident polarized light can give information about the preferential orientation of crystal planes. Figure 4 shows the FT-IR absorption spectra of $\mathrm{TiO}_{2}-300$ and $\mathrm{TiO}_{2}-600$ samples recorded with $s$-(perpendicular) and $p$-(parallel) polarized light. In this case only the spectral re-
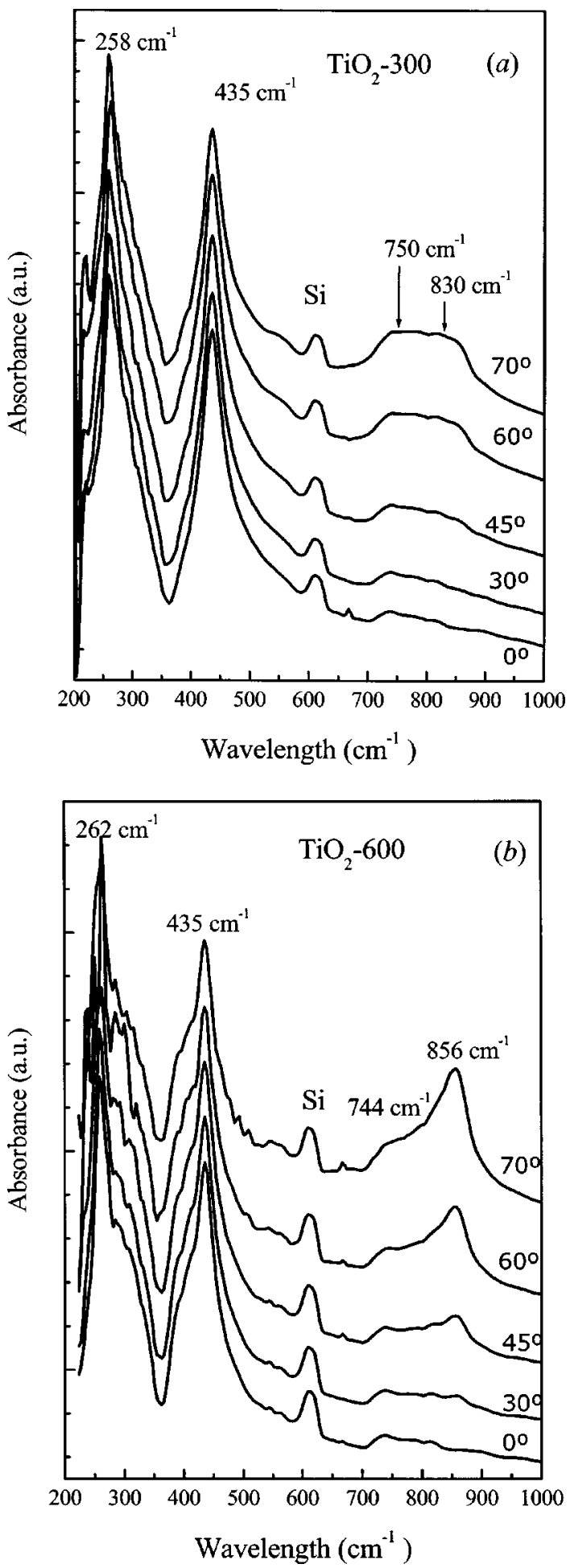

FIG. 3. Experimental absorbance of anatase thin layers prepared at $300{ }^{\circ} \mathrm{C}$ (a) and $600{ }^{\circ} \mathrm{C}$ (b) measured with unpolarized light. The band corresponding to the Si substrate has been marked.

gion from 400 to 1000 was accessible to the analysis due to the absorbance of the $\mathrm{KBr}$ polarizer at lower frequencies. The spectra of the $\mathrm{TiO}_{2}-300$ sample have the same aspect as those in Fig. 3 (taken with unpolarized light), either for parallel and perpendicular polarization. However, in the case of the sample $\mathrm{TiO}_{2}-600$, although the spectra for $s$ polarization do not depict any significant difference with the incidence 
TABLE IV. Vibrational IR modes of anatase.

\begin{tabular}{lcc}
\hline \hline Symmetry & $\begin{array}{c}\text { Transverse } \\
\text { frequency } \omega_{T}\left(\mathrm{~cm}^{-1}\right)\end{array}$ & $\begin{array}{c}\text { Longitudinal } \\
\text { frequency } \omega_{L}\left(\mathrm{~cm}^{-1}\right)\end{array}$ \\
\hline$E_{u}$ & 262 & 366 \\
$E_{u}$ & 435 & 876 \\
$A_{2 u}$ & 367 & 755 \\
\hline \hline
\end{tabular}

angle of the radiation, those for $p$ polarization show a net enhancement of the longitudinal mode at $856 \mathrm{~cm}^{-1}$, in a way similar to that found in the spectra with unpolarized light.

\section{DISCUSSION}

To interpret the absorbance of thin layers of anatase we have calculated the theoretical absorbance of anatase thin films deposited on silicon by the above described model by using its IR optical constants, measured by González et al. ${ }^{24}$ The calculated theoretical absorbance spectra corresponding to two different crystallite orientations and several different incidence angles for $p$-polarized light are displayed in Fig. 5. The spectra calculated for $s$-polarized light were omitted because they do not appreciably change with incidence angle. ${ }^{1}$ In the first case considered $c_{\text {pez }}=1 / 9$, the thin layer is anisotropic and composed of crystallites whose optical $c$ axes is preferentially tilted $90^{\circ}$ to the normal surface vector. In this case, two large maxima of absorbance with small shoulders are present at 242 and $440 \mathrm{~cm}^{-1}$ corresponding to the transverse optical (TO) $E_{u}$ phonons $^{24}\left(258\right.$ and $\left.435 \mathrm{~cm}^{-1}\right)$. At high values of incidence angle, an absorbance band ranging from 750 to $900 \mathrm{~cm}^{-1}$ is clearly visible. The maximum of this band shifts from 835 to $875 \mathrm{~cm}^{-1}$ for higher values of incidence angle. This final value corresponds to the highest frequency longitudinal $E_{u}$ phonon $\left(876 \mathrm{~cm}^{-1}\right)$.

In the case where the crystallites are randomly oriented, $c_{\text {pez }}=1 / 3$, it can be seen that the general aspect of the absorbance curves in the region of transverse modes are similar to those of $c_{\mathrm{pez}}=1 / 9$. However, in the spectral area of longitudinal frequencies corresponding to high incidence angle spectra, the maximum of the longitudinal $E_{u}$ phonon (876 $\mathrm{cm}^{-1}$ ) is now flanked by the longitudinal $A_{2 u}$ mode (755 $\mathrm{cm}^{-1}$ ), which now appears as a shoulder at $800 \mathrm{~cm}^{-1}$.

In Fig. 5 we have also plotted the absorbance curves corresponding to $c_{\text {pez }}=7 / 9$, which represents the case of crystallites with its $c$ axes preferentially orientated parallel to the normal of the surface sample. In this situation, the spectral region from 200 to $600 \mathrm{~cm}^{-1}$ displays two strong absorbance bands corresponding to the $E_{u}$-TO modes. However, the high frequency band corresponding to the longitudinal optical (LO) phonons is different. In this case, the $A_{2 u}$ mode $\left(755 \mathrm{~cm}^{-1}\right)$ is similar or even more prominent than the $E_{u}$ $\left(876 \mathrm{~cm}^{-1}\right)$ especially in case of the largest incidence angles.

The use of Table I in addition to a close observation of the spectral region corresponding to the LO modes could help to determine if an anisotropic polycrystalline sample is textured. In the case that $c_{\text {pez }}<1 / 3$ we see in Table I that along the $z$ axis the dielectric tensor takes the value of the ordinary dielectric constant. It implies that at higher incidence angles and with parallel polarization, only the LO fre- quencies of modes of degeneracy 2 could be visible (i.e., $E_{u}$ modes). If $c_{\mathrm{pez}}=1 / 3$, the sample is isotropic and under both parallel and perpendicular polarization the same spectra should be obtained at grazing angles. Finally, when $c_{\text {pez }}$ $>1 / 3$ the spectral area corresponding to the LO modes measured at high incidence angles and under parallel polarization are dominated by the longitudinal extraordinary modes. This simple scheme agrees very well with the experimental data. For the sample prepared at $300{ }^{\circ} \mathrm{C}$ and measured at high incidence angles with the polarized light (Fig. 4), bands around $800 \mathrm{~cm}^{-1}$ appear similar to those of theoretical spectra calculated for $c_{\mathrm{pez}}=1 / 3$. In this sample no indication of anisotropy was found since the spectra recorded with both the parallel and perpendicular components of the polarized spectra were similar [Figs. 4(a) and 4(c)]. On the contrary, the sample prepared at $600{ }^{\circ} \mathrm{C}$, parallel and perpendicular polarized spectra are no longer similar. In this case, the composite exhibits a true anisotropic behavior. The shape of the LO band indicates that the $E_{u}\left(876 \mathrm{~cm}^{-1}\right)$ mode is active at higher incidence angles in agreement with a partial orientation of the sample corresponding to $c_{\text {pez }}<1 / 3$.

Spectra taken with unpolarized light (Fig. 3), are experimentally easier to record, but cannot be used to perform quantitative analysis because they are a linear combination of parallel and perpendicular components. However, from a qualitative point of view, it should be noted that unpolarized spectra at higher angles $\left(60^{\circ}\right.$ and $\left.70^{\circ}\right)$ (Fig. 3) look similar to those taken with the parallel component of the electric field but at a lower angle $\left(50^{\circ}\right)$ [Figs. 4(a) and 4(b)]. Thus, it can be concluded that unpolarized spectra taken at near grazing angles have qualitative but valuable information about textures in the region of longitudinal modes in a way similar to the parallel polarized spectra.

According to our interpretation of IR spectra, it seems that $c$ axes of crystallites of the $\mathrm{TiO}_{2}-600$ sample present a certain preferential orientation parallel to its external surface, a fact that is corroborated by x-ray diffraction results. This orientation corresponds to a preferential growth of (101) planes parallel to the surface. The crystallographic $c$ axis of the anatase structure $(a=3.776$ and $c=9.486 \AA)$ forms an angle of $\phi_{(101)}=21.70^{\circ}$ with respect to the (101) plane. Therefore, in the oriented (101) $\mathrm{TiO}_{2}-600$ sample, the $c$ axes of the crystallites describe a cone-shaped distribution around the azimutal axes perpendicular to the sample, being $\alpha_{\text {th }}$ the angle between the axis and the generatrix of the cone. According to Eq. (18), and taking $\alpha_{\mathrm{th}}=90^{\circ}-\phi_{(101)}=68.28^{\circ}$, we get $c_{\text {pez }}=0.07$. This value is close to $c_{\text {pez }}=1 / 9=0.11$, whose IR theoretical spectra are represented in Fig. 4(a).

In order to get a closer view of the validity of the method, we have plotted together (Fig. 6) the experimental and calculated absorbance spectra ( $p$ polarization) of anatasa films for different incidence angles. The experimental plotted spectra correspond to samples heated at 300 and $600{ }^{\circ} \mathrm{C}$ and the theoretical ones to those calculated assuming that crystallites are oriented according to values of $c_{\text {pez }}, 1 / 9$ and $1 / 3$ (random distribution). A direct comparison of the spectra reveals the advantages and weaknesses of the proposed method are clearly visible. Firstly, it should be noted that calculated spectra follow the same general trend as the experimental 

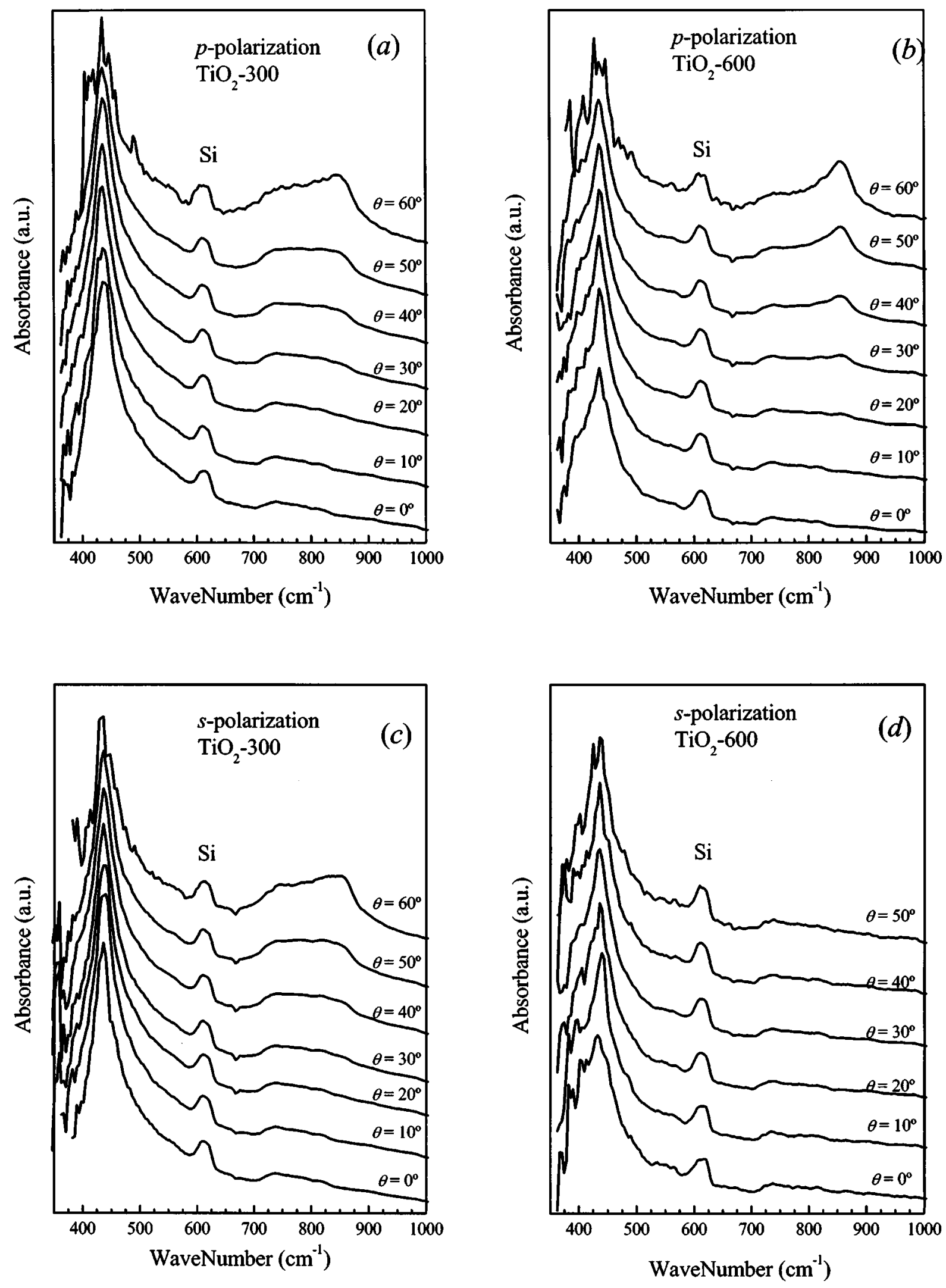

FIG. 4. Experimental absorbance of anatase thin layers prepared at $300{ }^{\circ} \mathrm{C}$ (a) and (c) and $600{ }^{\circ} \mathrm{C}$ (b) and (d) measured with parallel (a) and (b) and perpendicular light (c) and (d). The band corresponding to the Si substrate has been marked. 

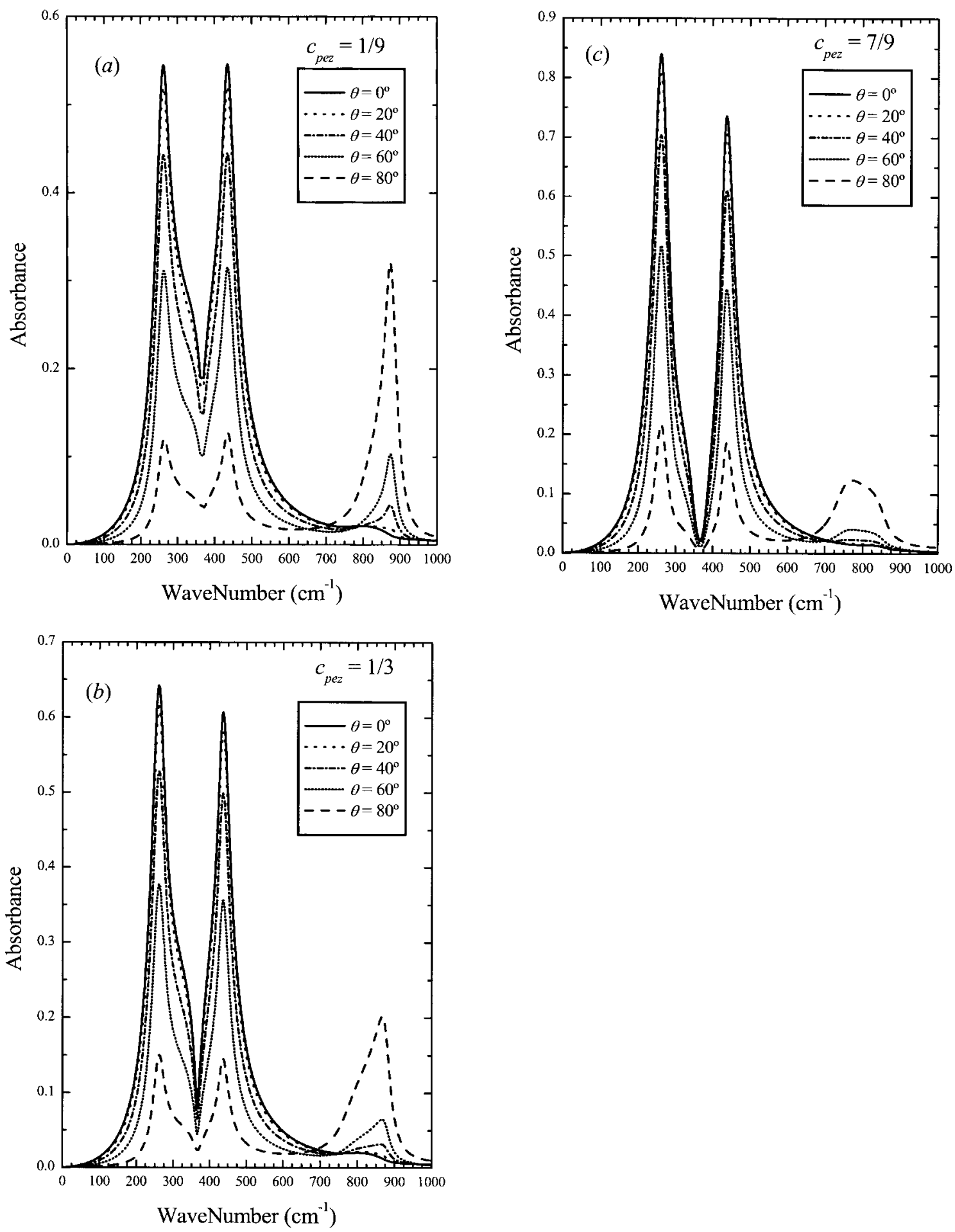

FIG. 5. Calculated values of absorbance for $L_{p}=1 / 3$ and $c_{\mathrm{pez}}=1 / 9$ (a), $c_{\mathrm{pez}}=1 / 3$ (b), and $c_{\mathrm{pez}}=7 / 9$ (c).

ones. However, discrepancies between them appear around 610 and $750 \mathrm{~cm}^{-1}$. These bands are due to multiphonon processes of the silicon substrate. ${ }^{25}$ In the same way, the longitudinal frequency of calculated spectra is shifted 25 $\mathrm{cm}^{-1}$ to higher frequencies in both kinds of samples. The origin of this mismatch remains unclear and we attribute it to absorbance fluctuations versus frequency of the silicon substrate in this spectral area, ${ }^{25}$ or more likely, to a possible error in the fitted longitudinal frequency measured on the anatase single crystal. ${ }^{24}$ In this sense, it should be noted that the calculated maximum of the high incidence angle curve exactly coincides with the longitudinal frequency of the anatase single crystal. Thus, in order to increase the accuracy of this method, in the future it will be necessary to properly characterize the substrate and to know with good precision the IR refractive index of the deposited film.

\section{CONCLUSIONS}

In this article we have shown that longitudinal phonon modes measured by absorption infrared spectroscopy at grac- 

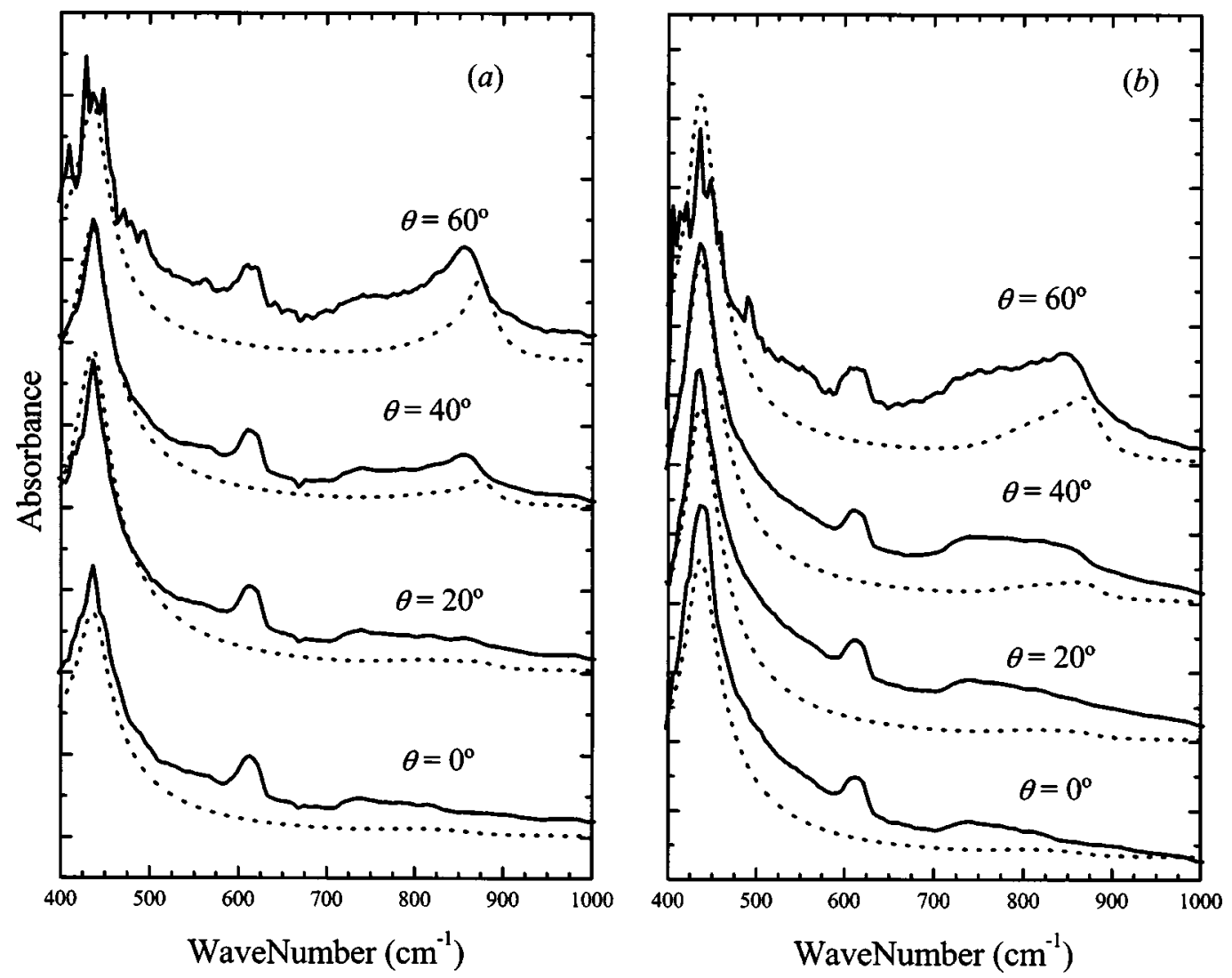

FIG. 6. Experimental (continuous line) absorbance curves of anatase thin films prepared at $600{ }^{\circ} \mathrm{C}$ (a) and $300{ }^{\circ} \mathrm{C}$ (b) and calculated (--) for $L_{p}=1 / 3$ and $c_{\text {pez }}=1 / 9(\mathrm{a}), c_{\mathrm{pez}}=1 / 3(\mathrm{~b})$, at different incidence angles for $p$-polarized light.

ing angles can be used to determine the presence of textures in thin layers of uniaxial materials. A simple theoretical model has been introduced and it has been shown that it reproduces quite well all the features of these spectra. Texture information obtained from IR spectroscopy was similar to that afforded by conventional XRD treatment, which support the validity of our IR model. Therefore, IR spectroscopy could be used as a valuable quick test to detect the presence of textures in dense anisotropic samples.

\section{APPENDIX A: VALIDITY OF THE EFFECTIVE MEDIUM APPROXIMATION FOR ANISOTROPIC COMPOSITES}

The aim of this section is to describe the model employed to determine the effective dielectric tensor in heterogeneous materials composed of an isotropic phase (labeled as " $m$ ") and uniaxial crystallites (labeled as " $p$ "). According to previous works, ${ }^{12,9}$ the average dielectric function along the direction of the external electric field $\mathbf{E}_{0}$ of an heterogeneous system can be expressed as

$$
\begin{aligned}
\left\langle\in{ }_{0} \boldsymbol{\epsilon}\right\rangle_{\mathbf{E}_{0}} & =\frac{\iiint_{V} \mathbf{D}_{\mathbf{E}_{0}}(\mathbf{r}) d^{3} \mathbf{r}}{\iiint_{V} \mathbf{E}_{\mathbf{E}_{0}}(\mathbf{r}) d^{3} \mathbf{r}}, \\
\in{ }_{0}\langle\boldsymbol{\epsilon}\rangle_{\mathbf{E}_{0}} & =\frac{(1-f)\left\langle\mathbf{D}_{m}\right\rangle_{\mathbf{E}_{0}}+f\left\langle\mathbf{D}_{p}\right\rangle_{\mathbf{E}_{0}}}{(1-f)\left\langle\mathbf{E}_{m}\right\rangle_{\mathbf{E}_{0}}+f\left\langle\mathbf{E}_{p}\right\rangle_{\mathbf{E}_{0}}},
\end{aligned}
$$

where, $\langle D\rangle_{\mathbf{E}_{0}}$ and $\langle E\rangle_{\mathbf{E}_{0}}$ are, respectively, the space average of the displacement and electric field in the direction of the external applied field $\mathbf{E}_{0}$ and $\boldsymbol{\epsilon}_{0}$ is the vacuum permittivity. These expressions are valid when the particle size is much smaller than the incident wavelength radiation. In order to estimate these averages it has been assumed that individual particles are ellipsoids, whose geometric axes are parallel to the optical ones and that the external field around these particles is homogeneous (effective medium hypothesis).

To determine the effective dielectric constant as it appears in Eq. (A1) it is necessary to relate the internal particle field $\mathbf{E}_{p}$ with the external average value $\langle E\rangle_{\mathbf{E}_{0}}$. The link between both fields, determined by the Poisson equation corresponding to a spheroid of dielectric tensor $\boldsymbol{\epsilon}_{p}$ embedded in an anisotropic matrix, is characterized by its dielectric tensor $\langle\boldsymbol{\epsilon}\rangle$ under the effect of an external and homogeneous electric field. We will assume that the heterogeneous medium presents uniaxial behavior. This simplification allows us to describe the dielectric tensor by only two components: $\left\langle\epsilon_{\|}\right\rangle$and $\left\langle\epsilon_{\perp}\right\rangle$, i.e., the effective dielectric constant in the direction parallel and perpendicular of the average field respectively. According to this reference frame, the dielectric tensor is given by

$$
\tilde{\epsilon}(\theta)=\left(\begin{array}{cc}
\left\langle\epsilon_{\|}\right\rangle-\Delta \epsilon \sin ^{2} \theta & \langle\Delta \epsilon\rangle \sin \theta \cos \theta \\
\langle\Delta \epsilon\rangle \sin \theta \cos \theta & \left\langle\epsilon_{\|}\right\rangle-\langle\Delta \epsilon\rangle \cos ^{2} \theta
\end{array}\right),
$$


where $\theta$ is the angle of the ray direction in respect to the optical axis of the crystallite, and $\langle\Delta \epsilon\rangle$ is given by

$$
\langle\Delta \epsilon\rangle=\left\langle\epsilon_{\|}\right\rangle-\left\langle\epsilon_{\perp}\right\rangle .
$$

In order to simplify the expressions, we will assume that the anisotropic particles have a spherical shape. The solution of the Poison equation, applied to a spherical inclusion inside an anisotropic matrix can be approximated by

$$
V(r, \theta)=\left\{\begin{array}{ll}
E_{i} r \cos \theta+\Delta V_{i}(r, \theta) & r<a \\
\langle E\rangle r \cos \theta+\mu \frac{\cos \theta}{r^{2}} & r>a
\end{array} .\right.
$$

This potential is similar to that of the isotropic case except for the term $\Delta V_{i}$, which takes into account the effect of the anisotropy of the space outside the sphere in it. This potential will induce an electric field $\Delta E_{i}$. By applying boundary conditions into the sphere surface $(r=a)$ for the electric and displacement field we get

$$
\begin{aligned}
& \left.E_{i}\right|_{t, r=a}=\left.E_{e}\right|_{t, r=a}, \\
& \left.D_{i}\right|_{n, r=a}=\left.D_{e}\right|_{n, r=a}, \\
& \frac{\Delta E_{i}}{E_{i}}=\frac{\langle\Delta \epsilon\rangle}{\left\langle\epsilon_{\|}\right\rangle} \sin ^{2} \theta\left(\frac{\mu}{a^{3}}-2\langle E\rangle\right) .
\end{aligned}
$$

Thus, the contribution of $\Delta E_{i}$ to the average electric field parallel to $\mathbf{E}_{i}$ is cancelled due to the symmetry of the field distribution

$$
\left\langle\Delta E_{i}\right\rangle=\int_{V_{p}} \frac{\langle\Delta \epsilon\rangle}{\left\langle\epsilon_{\|}\right\rangle} E_{i}\left(\frac{\mu}{a^{3}}-2\langle E\rangle\right) \sin ^{2} \theta \cos \theta d V=0 .
$$

This latter expression indicates that, at least at the first order, the component of the electric field inside a spherical inclusion in the direction of the external applied field is not modified by the anisotropy of the external medium. This result allows us to extend the results of the effective medium approximation from isotropic to anisotropic media. Thus, the expressions previously obtained for isotropic heterogeneous media also apply for each $l$ direction of an anisotropic composite [Eq. (1)].

Under these approximations the internal electric field for a single particle of the $k$ phase ( $p$ or $m$ ) along the $o$ direction is constant and given by

$$
E_{k o}=\frac{\langle\epsilon\rangle_{o}}{\left(1-L_{k o}\right)\langle\epsilon\rangle_{o}+L_{k o} \epsilon_{k o}}\langle E\rangle_{o},
$$

where $L_{k l}$ is the $l$ th depolarization factor of a $k$ ellipsoid. The space averaged field, parallel to the applied field along the $o$ direction turns out to be

$$
\begin{aligned}
\left\langle E_{o l}\right\rangle_{\mathbf{E}_{0}} & =\frac{1}{V} \int_{V} E_{o l}(\mathbf{r}) \gamma_{k l, \mathbf{E}_{0}}(\theta) d \mathbf{r} \\
& =\sum_{k=1}^{N} f_{k} E_{o k l} \int_{\Omega} \gamma_{k l, \mathbf{E}_{0}}(\theta) d \Omega=4 \pi \sum_{k=1}^{N} f_{k} E_{o k l} c_{k l},
\end{aligned}
$$

where $f_{k}$ is the filling factor of the $k$ phase, and $\gamma_{k l, \mathbf{E}_{0}}(\theta)$ is the density function corresponding to the probability of finding that the $l$ axis of the ellipsoid makes an angle $\theta$ with $\mathbf{E}_{0}$. In this sense $c_{k l}$ is the orientation average of the projection of the internal field to the direction of the external applied field

$$
\left.c_{k l}\right|_{\mathbf{E}_{0}}=\frac{1}{4 \pi} \int_{0}^{2 \pi} d \varphi \int_{0}^{\pi} \gamma_{k l, \mathbf{E}_{0}}(\theta) \sin \theta d \theta .
$$

The same calculation applies for the average displacement

$$
\begin{aligned}
\left\langle D_{o l}\right\rangle_{\mathbf{E}_{0}} & =\frac{1}{V} \int_{V} D_{o l}(\mathbf{r}) \gamma_{k l, \mathbf{E}_{0}}(\theta) d \mathbf{r} \\
& =\sum_{k=1}^{N} f_{k} \boldsymbol{\epsilon}_{o k l} E_{o k l} \int_{\Omega} \gamma_{k l, \mathbf{E}_{0}}(\theta) d \Omega \\
& =4 \pi \sum_{k=1}^{N} f_{k} \epsilon_{o k l} E_{o k l} c_{k l} .
\end{aligned}
$$

Finally, we get

$$
\langle\epsilon\rangle_{l}=\frac{\sum_{k=1}^{N} f_{k} \Sigma_{o=1}^{3} \frac{c_{k o l} \epsilon_{k o}}{\left(1-L_{k o}\right)\langle\epsilon\rangle_{l}+L_{k o} \epsilon_{k o}}}{\sum_{k=1}^{N} f_{k} \Sigma_{o=1}^{3} \frac{c_{k o l}}{\left(1-L_{k o}\right)\langle\epsilon\rangle_{l}+L_{k o} \epsilon_{k o}}} .
$$

\section{APPENDIX B}

In this Appendix we want to show the explicit expressions derived from the " $4 \times 4$ matrix transfer procedure" used in this work as described by Schubert et al. ${ }^{21-23}$ These expressions have been employed to derive the theoretical absorbance curves plotted in Figs. 5(a)-5(c). The relationship between the incidence, reflected and transmitted beam depend on the geometric configuration of the samples ${ }^{21}$ according to

$$
\left(\begin{array}{c}
A_{s} \\
B_{s} \\
A_{p} \\
B_{p}
\end{array}\right)=\mathbf{T}\left(\begin{array}{c}
C_{s} \\
0 \\
C_{p} \\
0
\end{array}\right)
$$

where $A_{s}, A_{p}, B_{s}, B_{p}, C_{s}$, and $C_{p}$ are the complex amplitudes of the parallel and perpendicular modes of the incident, reflected, and transmitted waves, respectively. $\mathbf{T}$ is the transfer matrix, which can be written as the product of the incident, exit and layer matrix

$$
\mathbf{T}=\mathbf{L}_{a}^{-1} \prod_{k=1}^{N} \mathbf{T}_{p k}^{-1} \mathbf{L}_{f}
$$

In our case we only have a single layer $(N=1)$. The values of the matrices for a thin film of an uniaxial layer of thickness $d$, are given by ${ }^{21}$ 


$$
\begin{aligned}
& \mathbf{T}_{p}^{-1}=\left(\begin{array}{cccc}
\cos \left(k_{0} N_{x z} d\right) & 0 & 0 & -i \frac{N_{x z}}{\epsilon_{x}} \sin \left(k_{0} N_{x z} d\right) \\
0 & \cos \left(k_{0} N_{y y} d\right) & -\frac{i}{N_{y y}} \sin \left(k_{0} N_{y y} d\right) & 0 \\
0 & i N_{y y} \sin \left(k_{0} N_{y y} d\right) & \cos \left(k_{0} N_{y y} d\right) & 0 \\
-i \frac{\epsilon_{x}}{N_{x z}} \sin \left(k_{0} N_{x z} d\right) & 0 & 0 & \cos \left(k_{0} N_{x z} d\right)
\end{array}\right), \\
& \mathbf{L}_{a}^{-1}=\frac{1}{2}\left(\begin{array}{cccc}
0 & 1 & \frac{-1}{n_{i} \cos \theta_{i}} & 0 \\
0 & 1 & \frac{1}{n_{i} \cos \theta_{i}} & 0 \\
\frac{1}{\cos \theta_{i}} & 0 & 0 & \frac{1}{n_{i}} \\
\frac{-1}{\cos \theta_{i}} & 0 & 0 & \frac{1}{n_{i}}
\end{array}\right) \text {, }
\end{aligned}
$$$$
t_{p}=\left(\frac{C_{p}}{A_{p}}\right)_{A_{s}=0}=\frac{1}{T_{33}} \quad t_{p}=\left(\frac{C_{p}}{B_{p}}\right)_{A_{s}=0}=\frac{1}{T_{43}} .
$$

$$
\mathbf{L}_{f}=\left(\begin{array}{cccc}
0 & 0 & \cos \theta_{s} & 0 \\
1 & 0 & 0 & 0 \\
-n_{s} \cos \theta_{s} & 0 & 0 & 0 \\
0 & 0 & n_{s} & 0
\end{array}\right),
$$

where $k_{0}=2 \pi / \lambda$ is the wave vector, $\epsilon_{x}$ and $\epsilon_{z}$ are the relative dielectric constant of the layer perpendicular and parallel to the normal to the plane, $n_{i}$ and $n_{s}$ are the refractive index of the incident medium and substrate, and $\theta_{i}$ and $\theta_{s}$ are the incidence and transmitted angles. Finally, the terms $N_{x x}$, $N_{y y}$, and $N_{x z}$ are defined following Ref. 21

$$
\begin{aligned}
& N_{x x}=N_{y y}=\sqrt{\epsilon_{x}-\epsilon_{i} \sin ^{2} \theta_{i}}, \\
& N_{y y}=\sqrt{\epsilon_{x}-\frac{\epsilon_{i} \epsilon_{x}}{\epsilon_{z}} \sin ^{2} \theta_{i} .}
\end{aligned}
$$

The final product $\mathbf{T}$ has all coefficients equal to zero except for $T_{11}, T_{21}, T_{33}$, and $T_{43}$.

$$
\mathbf{T}=\mathbf{L}_{a}^{-1} \mathbf{T}_{p k}^{-1} \mathbf{L}_{f}=\left(\begin{array}{cccc}
T_{11} & 0 & 0 & 0 \\
T_{21} & 0 & 0 & 0 \\
0 & 0 & T_{31} & 0 \\
0 & 0 & T_{43} & 0
\end{array}\right)
$$

Thus, the transmission and reflection coefficients, parallel and perpendicular are given by ${ }^{19}$

$$
\begin{aligned}
& \left(\begin{array}{c}
A_{s} \\
B_{s} \\
A_{p} \\
B_{p}
\end{array}\right)=\left(\begin{array}{cccc}
T_{11} & 0 & 0 & 0 \\
T_{21} & 0 & 0 & 0 \\
0 & 0 & T_{31} & 0 \\
0 & 0 & T_{43} & 0
\end{array}\right)\left(\begin{array}{c}
C_{s} \\
0 \\
C_{p} \\
0
\end{array}\right), \\
& t_{s}=\left(\frac{C_{s}}{A_{s}}\right)_{A_{p}=0}=\frac{1}{T_{11}} \quad r_{s}=\left(\frac{C_{s}}{B_{s}}\right)_{A_{p}=0}=\frac{1}{T_{21}},
\end{aligned}
$$

Finally the optical absorbance is given by

$$
\mathcal{A}_{s, p}=-\log _{10}\left(\left|t_{s, p}\right|^{2}\right) \text {. }
$$

${ }^{1}$ D. W. Berreman, Phys. Rev. 130, 2193 (1963).

${ }^{2}$ H. D. Riccius, J. Appl. Phys. 19, 4381 (1968).

${ }^{3}$ F. Proix and M. Balkanski, Phys. Status Solidi 32, 119 (1969).

${ }^{4}$ H. Poulet and J. P. Mathieu, Vibration Spectra and Symmetry of Crystals (Gordon and Breach, New York, 1976), p. 238.

${ }^{5}$ S. Hayashi, N. Nakamori, and H. Kanamori, J. Phys. Soc. Jpn. 46, 176 (1979).

${ }^{6}$ I. L. Skryabin, A. V. Radchik, P. Moses, and G. B. Smith, Appl. Phys. Lett. 70, 2221 (1997).

${ }^{7}$ M. Schubert, B. Rheinländer, E. Franke, H. Neumann, T. E. Tiwald, J. A. Woollam, J. Hahn, and F. Richter, Phys. Rev. B 56, 13306 (1997).

${ }^{8}$ E. Franke, M. Schubert, H. Neumann, T. E. Tiwald, D. W. Thompson, J. A. Woollam, J. Hahn, and F. Richter, J. Appl. Phys. 82, 2906 (1997).

${ }^{9}$ C. Pecharromán and J. E. Iglesias, Appl. Spectrosc. 54, 634 (2000).

${ }^{10}$ F. Yubero, M. Ocaña, A. Justo, L. Contreras, and A. R. Gonzalez-Elipe, J. Vac. Sci. Technol. A 18, 2244 (2000).

${ }^{11}$ J. P. Espinós, A. Fernández, A. Caballero, V. M. Jiménez, J. C. SánchezLópez, L. Contreras, D. Leinen, and A. R. González-Elipe, Adv. Mater. 3, 219 (1997).

${ }^{12}$ C. Pecharromán and J. E. Iglesias, Phys. Rev. B 49, 7137 (1994).

${ }^{13}$ P. E. Sulewski, T. W. Noh, J. T. McWhirter, and J. Sievers, Phys. Rev. B 36, 5735 (1987).

${ }^{14}$ T. W. Noh, P. H. Song, and A. J. Sievers, Phys. Rev. B 44, 5459 (1991) and references in therein.

${ }^{15}$ D. A. G. Bruggerman, Ann. Phys. (Leipzig) 24, 636 (1935).

${ }^{16}$ R. Landauer, J. Appl. Phys. 23, 770 (1952)

${ }^{17}$ M. Born and E. Wolf, Principles of Optics, 6th ed. (Pergamon, Oxford, 1983).

${ }^{18}$ D. W. Berreman, J. Opt. Soc. Am. 62, 502 (1972).

${ }^{19}$ P. Yeh, Surf. Sci. 96, 41 (1980).

${ }^{20}$ M. Schubert, Thin Solid Films 313, 323 (1998).

${ }^{21}$ M. Schubert, T. E. Tiwald, and C. M. Herzinger, Phys. Rev. B 61, 8187 (2000)

${ }^{22}$ A. Kreuter, G. Wagner, K. Otte, G. Lippold, A. Schindler, and M. Schubert, Appl. Phys. Lett. 78, 195 (2001).

${ }^{23}$ M. Schubert, Phys. Rev. B 53, 4265 (1996).

${ }^{24}$ R. J. González, R. Zallen, and H. Berger, Phys. Rev. B 55, 7014 (1997).

${ }^{25}$ D. F. Edwards, in Handbook of Optical Constants of Solids, edited by E. D. Palik (Academic, Orlando, FL, 1985), pp. 547-569. 